\title{
Maghreb : des élites entre deux mondes
}

Maghreb: An Elite Between Two Worlds

Magreb: élites entre dos mundos

\section{Pierre Vermeren}

\section{OpenEdition}

\section{Journals}

Édition électronique

URL : http://journals.openedition.org/ries/1252

DOI : $10.4000 /$ ries. 1252

ISSN : 2261-4265

Éditeur

Centre international d'études pédagogiques

Édition imprimée

Date de publication : 1 septembre 2005

Pagination : 47-56

ISBN : 978-2-85420-564-0

ISSN : $1254-4590$

Référence électronique

Pierre Vermeren, « Maghreb : des élites entre deux mondes », Revue internationale d'éducation de Sèvres [En ligne], 39 | septembre 2005, mis en ligne le 17 novembre 2011, consulté le 01 mai 2019. URL http://journals.openedition.org/ries/1252 ; DOI : 10.4000/ries.1252 


\section{Maghreb : des élites entre deux mondes}

\section{Pierre Vermeren}

La formation des élites est un enjeu majeur au Maghreb, cette ancienne «Afrique du Nord» colonisée par la France que nous limiterons ici au Maroc, à l'Algérie et à la Tunisie. Elle fut un enjeu colonial à l'heure du déclassement des vieilles élites musulmanes par les administrateurs français. Elle devint un combat crucial pour les nationalistes quand il fallut préparer le départ des Français. Elle fut ensuite un impératif pour doter les États indépendants d'un encadrement intellectuel, politique et technique. Elle demeure aujourd'hui l'enjeu majeur qui peut arrimer le Maghreb à l'Europe et à la mondialisation, avec une double contrainte, éviter une régression vers les «identités meurtrières» (un islamisme non maîtrisé), et "un exode des cerveaux » qui viderait la région de ses forces vives.

Certains traits relevés à l'échelle planétaire semblent observables au Maghreb comme à l'état cristallin. Trois d'entre eux s'imposent. Au Maghreb, la «consécration des élites» par l'enseignement supérieur est devenue une nécessité mais elle n'intervient qu'au terme d'un processus de sélection et de reproduction sociale des élites extérieur au système universitaire. La discrimination par les langues, en particulier par le français, joue dans ce processus un rôle central, largement adossé à un «circuit» de formation préscolaire et scolaire privé. Enfin, en dépit de l'autonomisation de son enseignement supérieur, il faut insister sur l'extrême dépendance/intégration du système de formation des élites du Maghreb au système français, et plus largement européen voire nord-américain. Cette internationalisation de la formation des élites pose la question de leur «retour au pays» dans un système mondialisé concurrentiel.

\section{ENSEIGNEMENT D'EXCELLENCE : FACTEURS SOCIAUX ET POLITIQUES}

Les filières d'excellence sont aujourd'hui le moyen de consacrer les élites économiques et sociales dans les trois pays du Maghreb. C'est l'un des apports majeurs de la période coloniale, fût-ce à son corps défendant. Car c'est en s'emparant des formations et des diplômes universitaires français que les nationalistes maghrébins ont pu gagner la bataille de l'indépendance: ils leur ont permis de conquérir le leadership au sein de leur société, tout en acquérant la qualité d'interlocuteur reconnu par le colonisateur. Non seulement cet acquis n'a pas été remis en cause lors de l'indépendance, mais il a permis la 
constitution d'un modèle scolaire et universitaire méritocratique calqué sur le modèle français. La coopération a joué dans ce domaine un rôle essentiel et de long terme.

Dans les années soixante-dix, soit vingt ans après les indépendances de 1956-1962, les systèmes de formation du Maghreb ont gagné en autonomie: arabisation progressive des filières juridiques et littéraires après celle du baccalauréat, massification scolaire, nationalisation du corps enseignant versus départ des coopérants, création de filières scientifiques et élitistes sur place (ainsi la faculté de médecine de Casablanca après celle de Rabat). Le modèle de référence est demeuré français. Toutefois, une diversification des formations et des trajectoires étudiantes s'est amorcée sous l'influence croisée des enseignants proche-orientaux et des formations anglo-saxonnes et européennes (Espagne, Allemagne). Mais le principal héritage des années 1970 et 1980 est le découplage des systèmes nationaux d'enseignement entre filières d'excellence réservées aux happy few et universités et filières de masse (les «universités casernes »).

Cette scission des systèmes d'enseignement permet d'identifier assez précisément les formations élitistes d'excellence, très réduites quant à leurs effectifs. Au Maroc, la voie royale demeure celle des grandes écoles françaises (d'ingénieurs, et de plus en plus d'ingénieurs commerciaux), la médecine (sur place ou à l'étranger), mais aussi quelques formations économiques et juridiques de haut niveau. En Tunisie, les tendances sont identiques mais, à l'inverse du Maroc, le système est encadré depuis des décennies par l'État, qui permet à l'élite des bacheliers de suivre ces formations, au besoin sur fonds publics, tandis que les hauts cadres administratifs sont favorisés. Dans les deux cas, le passage par de grandes écoles françaises ou par des établissements d'excellence européens ou nord-américains est fortement valorisé. En Algérie, la nature de l'État, les richesses pétrolières et la guerre civile des années 1990 ont donné un autre visage aux filières d'élites. Les ingénieurs pétroliers et les hauts cadres militaires constituent le cœur des élites, même si médecins, juristes et commerciaux de plus en plus souvent, conservent leur valeur.

$\mathrm{Au} \mathrm{XX} \mathrm{XX}^{\mathrm{e}}$ siècle, les trois pays d'Afrique du Nord ont hérité du système français de formation universitaire des élites qu'elles ont plutôt conforté. Celuici est aujourd'hui bien en place, avec trois traits majeurs : un très fort dualisme, le poids essentiel des formations francophones et l'intégration au système international de formation des élites. Toutefois, les origines sociales, familiales, locales et religieuses restent déterminantes dans le filtrage des élites maghrébines. Ce n'est pas au système universitaire qu'il faut imputer ce biais essentiel, mais aux sociétés, pour lesquelles la conservation familiale des biens acquis et celle des hiérarchies en place ne sont pas négociables. La «démocratie des talents» et "l'impartialité de la puissance publique» ne sont nullement légitimées par les élites. Le pays le plus emblématique à cet égard est le Maroc, dont 
le système makhzénien (monarchique) est basé sur la permanence des structures et des allégeances.

Le Maghreb a connu une grande phase d'ouverture politique et sociale durant les années soixante, quand il fallait doter les administrations publiques de centaines de milliers de cadres et de fonctionnaires nationaux. À l'époque, carrières et fortunes ont pu se constituer en dehors des logiques sociales habituelles, au sein d'économies nationales étatisées. Mais cette population de cadres moyens et supérieurs s'est peu à peu transformée en bureaucratie nationale réservant à ses enfants les moyens et les voies de la relève des générations... et des fonctions. La méritocratie et l'ouverture ont donc cédé la place à un système de reproduction particulièrement dur aux plus faibles mais aussi aux classes moyennes. Les études sociologiques que nous avons menées sur ce point au Maroc et en Tunisie (en dépit des réels efforts de cet État) sont à cet égard sans appel ${ }^{1}$. C'est aussi grâce à la lutte contre cette reproduction scolaire et sociale des élites francophones occidentalisées que les islamistes maghrébins ont réussi à incarner l'esprit de révolution.

Si bien que la relève, qu'elle soit en formation ou déjà insérée dans le marché de l'emploi, est pour l'essentiel constituée par les descendants des élites formées dans les années soixante. Les fils et les filles relèvent leurs pères ou leurs oncles. Tandis que l'élite formée dans les années 1960 et 1970 provenait d'un syncrétisme entre vieilles élites musulmanes, promus du nationalisme et nouveaux diplômés, les élites en cours de formation confortent les hiérarchies au détriment de nouvelles «compétences». C’est le verrouillage de la méritocratie qui remet en question la «circulation des élites», si nécessaire à la respiration des sociétés.

Cela étant, de puissants traits de caractère nationaux subsistent dans les trois pays, qui se surimposent au double système de reconnaissance que nous venons d'évoquer.

Sur le marché de l'encadrement, les allégeances royales restent déterminantes au Maroc. Ce système est appelé «makhzénien» (monarchiste), «fassi» (de Fès, l'ancienne capitale impériale), ou encore «technocratique (les ingénieurs du Palais). Nulle élite ne peut durablement s'imposer au Maroc en dehors de ces cercles étroits, souvent consanguins ${ }^{2}$. En Algérie, l'ascendant historique de la caste militaire depuis la guerre d'indépendance est tel que l'Armée populaire nationale (APN) joue un rôle proche de celui du makhzen marocain. Le tropisme régional y est cependant très puissant (en faveur des berbères chaouis (des Aurès) par exemple, qui constituent l'essentiel du haut

\footnotetext{
1. Pierre Vermeren, La formation des élites marocaines et tunisiennes, La Découverte, 2002.

2. Dans la tradition arabe, le mariage du fils avec la fille de l'oncle (appelé «mariage arabe») est une vieille pratique anthropologique. Au XXe siècle, la régression de cette pratique n'enlève rien au caractère primordial des alliances matrimoniales pour la préservation ou la constitution de tout patrimoine (ou capital au sens bourdieusien).
} 
encadrement militaire). Enfin, en Tunisie, pays de quasi parti unique, les affiliations partisanes (davantage bureaucratiques qu'idéologiques) jouent un rôle majeur dans le déroulement de toute carrière. La nature actuelle du régime tunisien est telle que la soumission inconditionnelle au système est la condition impérative de toute réussite sociale, politique et économique.

Le rôle dévolu au système de formation des élites et son autonomie par rapport à la société est donc à fortement relativiser au Maghreb où demeurent prégnantes l'inter-connaissance et la reconnaissance, au point que toute entreprise solitaire de promotion ou de réussite sociale est pratiquement vouée à l'échec.

\section{ÉLITES MAGHRÉBINES, ÉLITES FRANCOPHONES}

La francophonie est l'un des traits majeurs des élites maghrébines. Certes, il existe des élites arabophones, religieuses, littéraires ou juridiques mais elles sont partout dominées par les élites francophones, notamment aux plans économique, social ou politique. Cela est vrai dans les trois pays, avec des nuances. En Tunisie, les élites partagent un réel bilinguisme franco-arabe sans équivalent. En Algérie, malgré les discours, la francophonie domine dans de larges secteurs de la population, notamment dans la classe moyenne urbaine mais plus encore parmi les élites, d'autant que la résistance culturelle kabyle dénie toute représentativité à l'arabe. Le Maroc est le moins arabophone (au sens arabe classique) et le moins francophone des trois pays. Toutefois, la bourgeoisie et les élites marocaines sont largement francophones, le français représentant la langue seconde mais parfois aussi la langue maternelle (comme en Algérie) dans les milieux les plus huppés.

Si bien qu'en dépit de l'arabisation des années 1970-1980, qui a accompagné le développement des filières de masse, les élites maghrébines sont toujours formées en français. Il y a même une forte corrélation entre une haute maîtrise du français et une réussite sociale et professionnelle accomplie. Certes, des tentatives ont été faites par les États et certaines familles pour élargir la gamme linguistique mais le tropisme francophone subsiste. Ainsi, la Tunisie a envoyé des centaines d'étudiants pendant plus de quinze ans en Allemagne, au Canada et aux États-Unis. Mais ces élites scientifiques germanophones ou anglophones sont inadaptées à leur retour, dans une société où l'on travaille en français et en arabe. Pire, la tentation est alors forte de rester dans le pays des études supérieures, ce qui nourrit l'exode des cerveaux. Enfin, le coût des études en Amérique du Nord (surtout aux États-Unis) est suffisamment exorbitant pour ne concerner que quelques centaines d'étudiants maghrébins. C'est aujourd'hui vers l'Espagne que se dirigent davantage d'étudiants algériens et marocains, ce qui accroît la faible part des élites hispanophones, tradition ancienne dans le cas du Maroc. 
Les élites maghrébines francophones sont souvent de haut niveau, contrepartie d'un système de formation extraordinairement pyramidal. En schématisant, on peut dire que ce sont entre un et deux jeunes Maghrébins sur dix qui obtiennent le baccalauréat. Parmi ceux-ci, moins d'un sur dix se hisse dans des filières d'excellence, celles qui garantissent un emploi à haute rémunération. Dans le cas extrême du Maroc, ce sont quelques centaines d'étudiants et diplômés de très haut niveau qui entrent annuellement dans ce qu'il convient d'appeler «l'élite marocaine » ${ }^{3}$. Le plus gros bataillon est fourni par la cohorte des huit cents élèves marocains de chaque promotion des lycées français du Maroc (notamment Descartes à Rabat et Lyautey à Casablanca). Toutefois, une diversification s'observe avec l'université maroco-américaine El Akhawein d'Ifrane, quoique ses enseignements aient réintégré le français. En Algérie et en Tunisie, la pyramide est moins «pointue» pour diverses raisons.

En Tunisie, l'État contribue activement, depuis Bourguiba, à la formation d'élites nationales bilingues de haut niveau. Les Tunisiens qui entrent à Polytechnique à Palaiseau (ou à Polytechique La Marsa) sortent des lycées pilotes publics, à l'inverse des polytechniciens marocains, souvent issus des lycées français. Toutefois, le système tunisien est impitoyable, puisqu'à tous les niveaux, il élimine les élèves en difficulté relative sans leur offrir une seconde chance. C'est donc au prix d'une formidable sur-sélection qu'est assurée la formation d'élites nationales de haut niveau.

C'est finalement en Algérie que le système est le moins centralisé, même si les critères de la sélection sont impitoyables. L'ancienne république socialiste a conservé des éléments méritocratiques et égalitaires. Toutefois, la crise et la guerre ont fait le lit d'initiatives privées au profit des enfants de la "nomenklatura», cette bourgeoisie étatique (pétrolière, commerciale et militaire). Aux multiples instituts et universités d'Algérie, dont le prestigieux Institut algérien de pétrole (IAP) reconverti en pôle de management IAP Corporate University, il faut maintenant ajouter les filières privées et internationales en pleine croissance.

Ce système de formation dual, élitiste et inégalitaire, est en place depuis un quart de siècle. Jusqu'aux années 1980, les études à l'étranger représentaient une alternative aux blocages nationaux pour des dizaines de milliers d'étudiants ${ }^{4}$. Toutefois, l'explosion du nombre des étudiants, et la mise en place de politiques sécuritaires (visa Schengen notamment) en Europe durant les années 1990, ont fortement entravé les études à l'étranger. Ce système a entraîné des frustrations et une contestation de plus en plus virulentes depuis les années 1980, contestation animée par les étudiants et les partis islamistes, au nom de l'authenticité arabo-islamique.

3. Ali Ben Haddou, Maroc: les élites du Royaume, L'Harmattan, Paris, 1997.

4. Cf. Valérie Simon, in bibliographie. 
Cette contestation pourrait cependant aboutir au résultat inverse de celui escompté. Ainsi, dans les années 1980, l'enseignement privé était interdit au Maghreb, seulement toléré au Maroc. Le plus souvent francophone et réservé aux couches aisées de la population, le privé apparaissait comme une trahison anti-nationale. Aujourd'hui, ces réticences ont disparu et la crise de l'enseignement, le chômage de masse et la mondialisation entraînent une vive croissance de l'enseignement privé. De la sorte, des centaines d'écoles privées, notamment pré-scolaires et primaires, fleurissent dans chacun des trois pays, afin d'inculquer aux jeunes enfants la maîtrise précoce d'une langue française (voire anglaise) si déterminante pour leur avenir. Les parents savent qu'elle est la clef de la réussite future de leurs enfants, quelles qu'en soient les conséquences sur les classes pauvres exclues du système.

Si bien qu'au Maghreb, on ne touche pas à l'arabisation de l'enseignement public pour des raisons politiques et idéologiques (il ne faut pas remettre en cause les options de l'indépendance), alors qu'explose l'enseignement privé francophone en faveur des classes moyennes et aisées. Cette fuite des classes supérieures vers l'enseignement privé, et si possible à l'étranger, traduit une crise profonde de ces sociétés. Elle est aggravée par l'inextricable problème linguistique dont pâtit le Maghreb, lié à la présence d'un plurilinguisme non maîtrisé de facto (diglossie de l'arabe ${ }^{5}$, concurrence des langues berbères et du français, dissociation entre langues maternelles et langues écrites).

La permanence d'élites francophones peut apparaître comme un élément de stabilité et d'ouverture. Mais les risques sociaux collatéraux sont lourds. Contraintes à de multiples stratégies d'évitement, les familles aisées confortent leurs positions, tandis que les classes moyennes se révoltent et que l'alphabétisation des classes populaires et l'efficience de leur formation de base ne sont pas résolues.

\section{LA RÉINSERTION DIFFICILE DES ÉLITES NATIONALES MONDIALISÉES}

Si l'internationalisation de la formation des élites maghrébines est une réalité ancienne, c'est aujourd'hui la réinsertion de ces élites nationales mondialisées dans leur pays qui pose problème. Une brève histoire de la formation des élites universitaires maghrébines montre une incontestable proximité, derrière le trompe-l'œil des réalités nationales. Certes, la colonisation a varié dans ses modalités, entre l'Algérie française et ses deux voisins sous protectorat. Il en va

5. La diglossie, c'est le dédoublement de la langue qui n'est pas la même à l'écrit et à l'oral. Les Maghrébins parlent une langue orale non codifiée (le darija), leur langue, à proprement parler maternelle et nationale, alors qu'ils utilisent une langue écrite savante commune aux différents pays ayant adopté cette langue en partage, le fosha. 
de même pour les choix opérés aux indépendances: pouvoir "éclairé» en Tunisie, socialisme en Algérie et monarchie de droit divin pro-occidentale au Maroc. Pourtant, par-delà ces divergences, entretenues par d'exécrables rapports bilatéraux, le partenariat a été maintenu avec l'ancien colonisateur. L'inertie des systèmes de formation et l'importance des élites formées «à la française» ont permis aux structures de formation de s'enraciner et de prospérer.

On retrouve au Maghreb la primauté française du baccalauréat, la coupure entre grandes écoles et universités, la prééminence des mathématiques. Les réformes françaises ont souvent été appliquées avec quelques années de retard (loi Edgar Faure). Certes, une autonomisation des universités maghrébines s'est opérée, mais elle a peu touché le modèle étroit de la formation des élites. L'arabisation et son corollaire de réformes ont frappé les disciplines littéraires et les grosses universités (lettres, sciences religieuses, économiques, juridiques...). Les élites et leurs institutions ont été préservées, ne serait-ce que par la nécessaire compatibilité des troisièmes cycles universitaires. Pour coopérer, participer à des programmes communs de recherches, obtenir des financements et former certains doctorants, les meilleures formations du Maghreb, modestes quant à leurs moyens matériels et humains, doivent travailler avec les opérateurs européens.

Si bien que les élites maghrébines gardent un puissant tropisme français, même s'il est partiellement diversifié. Cela ne signifie pas que la dualité des systèmes, préalablement soulignée, se retrouve mécaniquement au plan politique. Ainsi, la concurrence entre formations d'excellence et formations standard s'exerce davantage au détriment des «dominés des dominants", pour reprendre une terminologie bourdieusienne. L'ingénieur francophone formé dans une petite école d'ingénieurs du Maghreb souffre davantage de la concurrence des ingénieurs de conception formés en France que le diplômé lambda d'une faculté de sciences économiques. Ce dernier n'a que peu de chances d'intégrer le marché de l'emploi mais il le sait, il l'a anticipé et il se sent d'un autre monde. En revanche, l'effet de déclassement joue à plein pour le jeune médecin ou ingénieur frais émoulu qui voit lui échapper les emplois et les fonctions de responsabilité les plus rémunératrices au profit des «élites »... Il n’est pas fortuit que ce soient les facultés des sciences et de médecine qui animent la contestation islamiste depuis les années 1980, terreau des cadres et militants islamistes les plus actifs.

Toutefois, face à la grande crise économique, sociale et politique que le Maghreb traverse depuis les années 1980, des ajustements sont nécessaires. Après avoir tenté de faire plier les gouvernements vers plus d'arabisation, d'authenticité et de nationalisme religieux, les islamistes ont perdu la bataille, au moins à court terme. La répression policière tunisienne, la guerre civile en Algérie et une extrême surveillance au Maroc ont ruiné ces espoirs. Si bien que 
la jeunesse déclassée s'est mise à rêver d'Europe. Même dans la petite Tunisie que l'on dit prospère, la jeunesse diplômée est frappée par le chômage. Les "diplômés-chômeurs" (souvent très diplômés) représentent plus d'un demimillion de personnes au Maghreb. L'exil des compétences est sans alternative, malgré les nombreux obstacles qui se dressent sur leur route.

Mais avec la mondialisation, les écarts Nord-Sud qui s'aggravent en Méditerranée et la politique internationale de recrutement des cadres de la part des économies riches, ce sont aussi les «élites» maghrébines qui sont tentées par l'expatriation. Le retour au pays était un impératif moral, national et familial jusque vers 1980-85. Depuis, il est devenu une question personnelle. Les jeunes diplômés, après leur formation à l'étranger (en totalité ou bien en troisième cycle), effectuent des stages et des expériences professionnelles valorisantes, bien payées, souvent sans équivalent au Maghreb. Car les économies maghrébines sont sous-encadrées, sous-bancarisées et peu productives, ce qui pousse les jeunes compétences à faire leurs armes à l'extérieur. Mais une fois celles-ci effectuées, la question du retour paraît complexe.

Cela est particulièrement vrai pour les jeunes filles, qui constituent une part conséquente des élites formées depuis les années 1990, et qui aspirent à la tranquillité des relations de travail et des rapports hommes-femmes en Europe. Cela concerne aussi les jeunes gens sans attache influente dans leur ville ou dans leur pays d'origine, et donc promis à des carrières de second plan (firmes multinationales mises à part). Il en va de même pour de nombreux médecins qui apprécient l'opulence des hôpitaux européens, quand la médecine du Maghreb gère une pénurie qui relève de l'indigence ${ }^{6}$ (30\% d'assurés sociaux contre $99 \%$ en France).

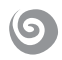

La question du retour des élites, mais aussi celle du départ des diplômés formés au Maghreb, confronte la région à l'un de ses plus grands défis. Il serait ici déplacé de formuler des recommandations pour faire évoluer ce complexe système de formation des élites maghrébines, qui combine des structures de formation autonomes et nationales à une puissante dépendance au système de formation francophone dont il partage les atouts et les insuffisances. En revanche, la coopération euro-maghrébine semble s’imposer pour rendre ce système plus acceptable par ces sociétés en voie de démocratisation, mais aussi plus efficient au vu des impératifs de développement économique et social.

6. Ce qualificatif est à appréhender au sens propre. Car au Maghreb, la crise hospitalière est si bien établie que la bourgeoisie et les couches moyennes optent systématiquement pour les cliniques privées payantes (en plein essor depuis quinze ans). Ainsi, au Maroc, 90 \% des patients de l'hôpital public sont munis du «certificat d'indigence ", qui leur donne accès à une gratuité théorique des soins (compte non tenu des médicaments, traitements, et des divers prélèvements monétaires illicites). 


\section{BIBLIOGRAPHIE}

BEN SALEM L. (1976) : "Développement et problème de cadres, le cas de la Tunisie », Cahiers du CÉRES, Série sociologique, $\mathrm{n}^{\circ}$ 3, Tunis.

BENRABAH M. (1999): Langue et pouvoir en Algérie, Histoire d'un traumatisme linguistique, Séguier, Atlantica, Biarritz.

GEISSER V. (coord.) (2001): Diplômés maghrébins d'ici et d'ailleurs: parcours migratoires et trajectoires d'insertion, CNRS-Éditions, Paris.

KADRI A. (1992): Le droit à l'enseignement et l'enseignement du droit, thèse de doctorat sous la direction du professeur Jean-Claude Passeron, soutenue le 3 avril 1992 à l'E.H.E.S.S.

KRICHEN A. (1986) : "La fracture de l'intelligentsia. Problèmes de la langue et de la culture nationales", in Tunisie au présent, Éditions du CNRS, pp. 297-341.

PERVILLÉ G. (1984): Les étudiants algériens de l'Université française 18801962, éditions du CNRS.

SIMON V. (1997): La migration des étudiants maghrébins en France et ses transformations (1962-1994), Université de Paris VII, 2 tomes.

VERMEREN P. (2001): Le Maroc en transition, La Découverte, Paris (rééd. Poche La Découverte 2002).

VERMEREN P. (2002): La formation des élites marocaines et tunisiennes, Des nationalistes aux islamistes, 1920-2000, Recherches La Découverte, Paris.

VERMEREN P. (2003) : Maghreb, la démocratie impossible?, Fayard. 
